MINI-SYMPOSIUM

\title{
Endothelial function and clinical outcome
}

J A Vita

Heart 2005;91:1278-1279. doi: 10.1136/hrt.2005.061333

U nder pro-atherosclerotic conditions, endothelial cells lose the ability to produce bioactive nitric oxide and demonstrate increased expression of vasoconstrictor, pro-inflammatory, and pro-thrombotic factors. The available evidence suggests that these alterations in endothelial phenotype contribute to the formation, progression, and rupture of atherosclerotic lesions. There currently is great interest in understanding the mechanisms and clinical relevance of these changes in endothelial cell biology, because they could lead to new approaches for the management of patients with atherosclerosis. While it is clear that the endothelium regulates many aspects of vascular homeostasis, current approaches to evaluate local "endothelial function" in humans have been limited to assessment of endothelium dependent vasodilation. In addition, investigators have the ability to measure blood concentrations of various endothelium derived thrombotic and inflammatory factors and, most recently, numbers of endothelial progenitor cells.

\section{ENDOTHELIAL DYSFUNCTION IN CORONARY ARTERIES}

The clinical relevance of endothelial dysfunction for cardiovascular disease events is strongly supported by studies in the human coronary circulation showing that impaired endothelium dependent vasodilation in response to acetylcholine or shear stress predicts clinical outcome. For example, more severe impairment of the vasomotor response to intracoronary acetylcholine infusion identifies individuals with increased risk for acute coronary syndromes, cardiovascular death, and stroke. ${ }^{1-4}$ Impaired flow mediated dilation ${ }^{2}$ and a more severe constrictor response to the cold pressor test ${ }^{25}$ also predict future cardiovascular events. These findings apply to patients with advanced coronary artery disease ${ }^{23}$ and to individuals with angiographically normal or nearly normal coronary arteries. ${ }^{135}$

\section{ENDOTHELIAL DYSFUNCTION IN PERIPHERAL ARTERIES}

Although studies in the coronary circulation have the greatest clinical relevance, it is interesting that measures of endothelial function in peripheral arteries also have predictive value for coronary heart disease. For example, impaired endothelium dependent dilation of forearm microvessels in response to acetylcholine predicts future events in patients with hypertension, ${ }^{6}$ stable coronary disease, ${ }^{7}$ or a recent acute coronary syndrome. ${ }^{8}$ Impaired flow mediated dilation of the conduit brachial artery predicts cardiovascular events in patients with hypertension, ${ }^{9}$ coronary artery disease, ${ }^{10}$ or peripheral arterial disease. ${ }^{11}$ It has been recently recognised that stiffness of the central aorta relates in part to the bioavailability of endothelium derived nitric oxide, ${ }^{12}$ and measures of aortic stiffness have also been shown to predict cardiovascular risk. ${ }^{13}$

In regard to circulating markers of endothelial dysfunction in peripheral blood, higher concentrations of soluble intercellular adhesion molecule-1 and plasminogen activator inhibitor are associated with increased cardiovascular risk, although other cell types may also produce these factors and the specific relevance of these findings to local endothelial function remains uncertain. Recent studies have suggested that the number of circulating endothelial progenitor cells relates to endothelium dependent vasodilation, ${ }^{14}$ but their relation to future cardiovascular disease events remains uncertain. Collectively, the studies of vasomotor function and circulating markers suggest that endothelial dysfunction may be a systemic state or that systemic factors have parallel effects in coronary and peripheral arteries.

Not all studies have shown that peripheral artery endothelial function is an independent predictor of future events after control for extent of atherosclerosis. A relatively small study by Neunteufl and colleagues showed that the predictive value of brachial artery flow mediated dilation was attenuated after control for the extent of coronary artery disease. ${ }^{10} \mathrm{~A}$ recent study also found that the relation between flow mediated dilation and events was no longer significant after extent of carotid intimal thickness was included in the multivariate model. ${ }^{15}$ In contrast, at least 15 studies in the coronary and peripheral circulations involving nearly 3000 patients have shown that endothelial dysfunction predicts events after adjusting for extent of coronary artery disease. ${ }^{3} 7$ The explanation for these apparently discrepant results remain unclear, but they could relate to differences in the cohorts studied or the specific techniques involved. Nevertheless, all of the available studies suggest a close relation between endothelial dysfunction and the atherosclerotic process. Moreover, a recent study by Modena and colleagues demonstrated that improvement in brachial artery flow mediated dilation following institution of antihypertensive treatment was associated with reduced cardiovascular risk compared to individuals that fail to improve. ${ }^{9}$ Although confirmatory studies are needed, this finding suggests that endothelial function might have utility as a surrogate marker with utility for evaluating risk reduction treatments.

\section{POSSIBLE MECHANISMS}

A number of potential mechanisms have been proposed to explain the relation between endothelial dysfunction and cardiovascular risk. For example, it is possible that endothelial dysfunction simply reflects the presence and extent of atherosclerosis, and it would not be surprising for patients with more extensive atherosclerosis to have a higher risk of cardiovascular events compared to patients with less or no atherosclerosis. The two published studies demonstrating a weakened correlation between endothelial dysfunction and cardiovascular events after adjustment for extent of disease $^{10}{ }^{15}$ support this potential mechanism. Similarly, it is possible that endothelial dysfunction represents the cumulative effects of traditional risk factors on the vasculature and that patients with a higher level of risk factors will be more likely to develop atherosclerosis and associated cardiovascular events. However, as discussed above, nearly all of the available studies suggest that endothelial dysfunction provides prognostic information beyond that provided by 
measuring the extent of atherosclerosis and the severity of traditional risk factors. Endothelial vasomotor function varies widely among patients with atherosclerosis, and many patients with advanced disease display endothelium dependent dilation comparable to that observed in healthy subjects. Thus, systemic endothelial dysfunction is not an inevitable consequence of atherosclerosis. Those patients with atherosclerosis and preserved endothelial function appear to have the lowest risk for events.

It is now well recognised that atherosclerosis is present and clinically silent for decades before plaque rupture and the development of acute coronary events. While many factors contribute to plaque vulnerability, the predictive value of endothelial dysfunction in patients with established atherosclerosis suggests that endothelial dysfunction might directly promote plaque rupture. A number of possible mechanisms might account for such an effect. For example, loss of endothelium dependent flow mediated dilation results in an inability to limit local shear stress at the arterial wall. Furthermore, stiffness of the central aorta has the potential to increase the pulsatility of coronary blood flow, and such alterations in hydrodynamic forces could increase the risk for plaque rupture or erosion. Loss of the anti-inflammatory effects of nitric oxide and increased endothelial expression of pro-inflammatory factors within the vasa vasorum of atherosclerotic conduit arteries could promote accumulation of inflammatory cells in plaque and the consequent vulnerability to rupture. Neovascularisation of plaque has also been related to plaque vulnerability, and pathological states associated with endothelial vasomotor dysfunction may also promote plaque angiogenesis. ${ }^{16}$

Abnormalities of the vascular endothelium also have the potential to exacerbate the consequences of plaque rupture. For example, loss of the antiplatelet effects of nitric oxide and prostacyclin and expression of pro-thrombotic and antifibrinolytic factors may promote arterial thrombosis and increase the likelihood that plaque rupture will lead to lumen obstruction. Loss of homeostatic effects of nitric oxide and other endothelium dependent vasodilators worsen the vasoconstrictor effects of thrombin, serotonin, and other factors produced at the site of arterial thrombosis. ${ }^{17}$ These effects in conduit coronary arteries and downstream resistance vessels may limit blood flow and increase the severity of myocardial ischaemia and/or infarction. Although many of these mechanisms remain speculative in human disease, there appears to be many potential mechanistic links between endothelial dysfunction and the pathogenesis of acute coronary syndromes.

\section{SUMMARY}

Abnormalities of endothelial function, particularly loss of endothelium dependent vasodilation, are associated with worse clinical outcome in individuals at risk for atherosclerosis and in patients with established disease. Despite the highly localised nature of plaque rupture in acute coronary syndromes, patients at risk for such events also display endothelial dysfunction in peripheral arteries. Blood concentrations of factors derived from activated endothelial cells and the number of circulating endothelial progenitor cells also may relate to cardiovascular risk. All of these findings strongly suggest that endothelial dysfunction contributes to the pathogenesis of cardiovascular disease events and that assessment of endothelial function could have clinical utility. However, translation of these findings to the care of individual patients still awaits the development of better and more standardised methodology for the assessment of endothelial function. In addition, appropriate prospective studies will be needed to demonstrate the incremental value of measuring endothelial function over and above the many other approaches that are available to evaluate cardiovascular risk.

\section{ACKNOWLEDGEMENTS}

Dr Vita's work is supported by grants from the US National Institutes of Health (HL60886, HL55993, M01RR00533, HL75795, HL76784, and HL70100).

Correspondence to: Joseph A Vita, MD, Section of Cardiology, Boston Medical Center, 88 East Newton Street, Boston, MA 021 18, USA; jvita@ bu.edu

\section{REFERENCES}

1 Suwaidi JA, Hamasaki S, Higano ST, et al. Long-term follow-up of patients with mild coronary artery disease and endothelial dysfunction. Circulation 2000;101:948-54.

2 Schachinger V, Britten MB, Zeiher AM. Prognostic impact of coronary vasodilator dysfunction on adverse long-term outcome of coronary heart disease. Circulation 2000;101:1899-906.

3 Halcox JP, Schenke WH, Zalos G, et al. Prognostic value of coronary vascular endothelial dysfunction. Circulation 2002;106:653-8.

4 Targonski PV, Bonetti PO, Pumper GM, et al. Coronary endothelial dysfunction is associated with an increased risk of cerebrovascular events. Circulation 2003; 107:2805-9

5 Schindler TH, Hornig B, Buser PT, et al. Prognostic value of abnormal vasoreactivity of epicardial coronary arteries to sympathetic stimulation in patients with normal coronary angiograms. Arterioscler Thromb Vasc Biol 2003;23:495-501.

6 Perticone F, Ceravolo R, Pujia A, et al. Prognostic significance of endothelial dysfunction in hypertensive patients. Circulation 2001;104:191-6.

7 Heitzer T, Schlinzig T, Krohn K, et al. Endothelial dysfunction, oxidative stress, and risk of cardiovascular events in patients with coronary artery disease. Circulation 2001; 104:2673-8

8 Fichtlscherer S, Breuer S, Zeiher AM. Prognostic value of systemic endothelial dysfunction in patients with acute coronary syndromes: further evidence for the existence of the "vulnerable" patient. Circulation 2004;110:1926-32.

9 Modena MG, Bonetti L, Coppi F, et al. Prognostic role of reversible endothelial dysfunction in hypertensive postmenopausal women. J Am Coll Cardiol 2002:40:505-10.

10 Neunteufl T, Heher S, Katzenschlager R, et al. Late prognostic value of flowmediated dilation in the brachial artery of patients with chest pain. Am J Cardiol 2000;86:207-10.

11 Gokce N, Keaney JF Jr, Hunter LM, et al. Predictive value of non-invasivelydetermined endothelial dysfunction for long-term cardiovascular events in patients with peripheral vascular disease. J Am Coll Cardiol 2003;41:1769-75.

12 Wilkinson IB, MacCallum H, Cockcroft JR, et al. Inhibition of basal nitric oxide synthesis increases aortic augmentation index and pulse wave velocity in vivo. Br J Clin Pharmacol 2002;53:189-92.

13 Boutouyrie P, Tropeano Al, Asmar R, et al. Aortic stiffness is an independent predictor of primary coronary events in hypertensive patients: a longitudinal study. Hypertension 2002;39:10-15.

14 Hill JM, Zalos G, Halcox JP, et al. Circulating endothelial progenitor cells, vascular function, and cardiovascular risk. N Engl J Med 2003;348:593-600.

15 Fathi $\mathbf{R}$, Haluska $B$, Isbel $N$, et al. The relative importance of vascular structure and function in predicting cardiovascular events. J Am Coll Cardiol 2004;43:616-23

16 Moulton KS, Vakili K, Zurakowski D, et al. Inhibition of plaque neovascularization reduces macrophage accumulation and progression of advanced atherosclerosis. PNAS 2003;100:4736.

17 Shimokawa H, Vanhoutte PM. Impaired endothelium-dependent relaxation to aggregating platelets and related vasoactive substances in porcine coronary arteries in hypercholesterolemia and atherosclerosis. Circ Res 1989;64:900-14. 